\title{
Improved TCP Prediction Congestion in Mobile Ad Hoc Network Based on Cross-Layer and Fuzzy Logic
}

\author{
https://doi.org/10.3991/ijim.v15i14.22021
}

\author{
Moulay Hicham Hanin ( $\left.{ }^{\varpi}\right)$, Mohamed Amani, Youssef Fakhri \\ Ibn Tofail University, Kenitra, Morocco \\ moulay.hicham.hanin@uit.ac.ma
}

\begin{abstract}
Mobile ad hoc network (MANET) is among the networks which do not require any infrastructure to put nodes in communication. Due to its own nature, it is used by several applications. Even though it's a network that is extremely challenging and mostly when TCP is applied. In this paper, we have proposed a new improvement in the TCP algorithm that employed fuzzy logic to predict packet loss and avoid congestion. Specifically, we have used tree metrics such as stability, energy, and signal strength to use in fuzzy logic systems. To accomplish our approach, we have established some modifications based on cross-layer. The results of the relevant simulation performed by NS3 demonstrated that our approach globally improves the performance of TCP in MANET. Precisely reduce the packet overhead and energy consumption also enhance throughput.
\end{abstract}

Keywords-MANET, MANET, TCP, AODV, DSDV, OLSR, Fuzzy Logic, congestion, Cross-layer

\section{$1 \quad$ Introduction}

Recently, mobile ad hoc networks (MANET) have shown a high growing interest to enhance the performance of protocols for supporting quality of service (QoS) in MANET. Ad Hoc network is a set of wireless mobile nodes that does not require any centralized administration to establish a communication. Due to its self-configuration, flexibility, and distributed nature, it is used in many applications such as military service, vehicular networks and disaster recovery. MANETs have many specifications, like multi-hop communication, dynamic topology, and limited resources. These characteristics make routing protocol design as particular challenges [1]. The principal objective is to maximize network lifetime and energy efficiency in MANET routing protocol, so that guarantees QoS and enhances performance of communication. To achieve these goals, several researches have been carried out. Three types of routing have been mainly defined in MANETs: reactive routing protocols, proactive routing and hybrid routing protocols.

An important feature in ad hoc networks is the ability to link to wired networks, in order to access Internet services through a gateway. The mobiles which play the role of gateways (most often all the mobiles) implement a router in their circuits, the mo- 
bility being managed by the Mobile IP protocol [2]. This group has taken the classic Internet protocols and refined them to work with mobile routers.

The core functionality of the transport layer is the transfer of end-to-end data from transmitter to receiver in a reliable and economical manner. It isolates the upper levels from technological variations and imperfections in the lower layers. It does this by employing transport protocols that use network layer (IP) services to deliver data. The protocols widely deployed in IP networks are TCP (Transmission Control Protocol) [3], UDP (User Datagram Protocol) [4], DCCP (Datagram Congestion Control Protocol) [5] and SCTP (Stream Control Transmission Protocol) [6].

Most TCP deployments have been carefully designed in the context of wired networks. In an ad hoc environment the implementation of TCP can lead to poor performance [7] due to the inherent properties of wireless networks. And this noticed in the last years, due to the rapid industrial development of various communication wireless devices technology of mobile edge computing (MEC). Several studies have been carried out to improve performance of MEC based on deep learning networks and especially what is oriented towards intelligent IoT [8][9]. Since the advent of the TCP transport protocol, several algorithms that aim to improve the congestion control mechanism have been proposed. All these algorithms use the same transmission principle as basic TCP; however, each algorithm offers a new mechanism for detecting and avoiding congestion. These different variants have been proposed with the aim of allowing TCP to react as well as possible to packet losses.

The main reasons that lead to a performance degradation in ad hoc networks come, as in wireless networks, from the quality of the wireless link, but also from the quality of the path. One reason for this degradation is the erroneous behavior of TCP, which mistakenly infers data loss as congestion and unnecessarily lowers its transmission rate. Therefore, the packets are dropped when the load on the network is greater than the capacity of the network in wired networks and which allow us to justify packet losses. On the other hand, in MANET networks congestion is not the only case caused by the loss of packets, there is stability, energy, link quality and interferences. At this level, the problem encountered by TCP in the MANET network is the enormous retransmission of lost packets.

Although TCP slows down sending massive packets by adjusting the window after the first declaration of lost packets due to various MANET's functional challenges like frequent link changes, hidden or exposed nodes, spatial reuse and incompatibility between TCP ACK packets and routing protocol. This justifies the large number of overhead packets in the MANET network. So, the best method of predicting congestion can improve the QoS of the TCP protocol.

The paper is organized as follows. In section 2, we have studied and analyzed articles that have improved the performance of MANET in order to use it to achieve a new contribution. In Section 3, we will present and dissect the process of our contribution. Simulation results are discussed and interpreted in section 4. In Section 5, Conclusion of our work and developed for the new research tracks.

The document you would like to format and import the styles. How this works depends very much on the version of MS WORD that you use. The styles' names to be 
used for online-journals.org are preceded by a "0_" which makes them appear first in the styles list and therefore easier to be found.

Now just place the cursor in the paragraph you would like to format and click on the corresponding style in the styles window (or ribbon).

\section{Related Work}

Much research focuses on improving the TCP protocol due to its congestion control mechanism, but it faces serious challenges when used in MANETs. Certainly, never succeeded in meeting all the challenges but they managed to minimize their impact.

The author Sumet and all [10] proposed a method based on the construction of optimization routes for the location of requests and minimize the wastage of limited resources, namely network bandwidth and node energy based on an increase in the size of control information to control routing packets in a heterogeneous network. The author chose to implement the method on a protocol that knows several limits in the case of dense networks. Hanin and al. proposed a Cross layer solution for improving the QoS of TCP in the Manets under the basis of different metrics [11]. The author calculates the metrics based on classical logic which does not give approximate values to the state of the metrics [12]. The work by Josh Kumar and A. Kathirvel addresses two main reasons that can reduce throughput in MANET which is the basis of link quality. The aim is to introduce an innovation called helper nodes which will help in the formation of alternate path and neutralize the ill effects, which provides a research impetus to increase the generalization decrease of packet loss. The author [13] in this article is giving a research impulse to increase generalization and gives a new practical classification. The work by authors in this article is giving a research impulse to increase generalization and gives a new practical classification.

In [14] the author tried to improve the duration of reliability in the ad hoc network based on probabilistic analysis to examine reliability metrics in node clustering. The method used scatter search in genetic algorithms which operated on a reference set to create new solutions. However, in this contribution, the author randomly chooses certain nodes to switch to their respective alternative clusters, which did not take into consideration the verification of the scalability of the nodes. Weimin Zheng et al. [15] proposed a congestion control practices finite time for the case of external disturbance algorithms. This contribution opens a track or researchers to exploit the control of the transmission and the active queue.

The conception of an efficient routing strategy and have a good scalability to integrate MANET with the internet is a very challenging problem, in this regard the author [16] suggest unpretentious integration scheme for a MANET and the wider Internet, based on the optimized link state routing (OLSR) To overcome the incompatibilities between different architectures. Precisely author redesigned and optimized routing Hello and TC messages to meet heterogeneity and gateway discovery needs without adding any additional messages. In another respect the author in [17] proposed an improvement in the quality of service based on the average end-to-end delay and 
packet loss, it cut down the delay experienced by data packets in the MANET network. in order to achieve 5G internet integration at MANETs with greater robustness and flexibility. Several routing protocols have been proposed for integrating MANET Network to the internet. Reference [18] considers that, with the aim of improving the performance of TCP to suit the routing requirements to over ad hoc systems.

MANET has limited resources and knows a high risk of congestion. like de congestion increases the probability of contention and losses MAC and TCP performance is severely degraded. To avoid network congestion, various techniques are followed. To improve the performance of TCP in Manet Suveg et al [19] have proposed a cross layer scheme which synchronizes the MAC and TCP layer. The idea is to dynamically adjust the TCP congestion window using the information received from the MAC layer contention window in order to, minimize the problem of overflow of the congestion window.

Handling the congestion efficiently attracted the attention of many authors [20] [21] [22]. In [23] The author proposed an idea based on Round-Trip Time (RTT) and bandwidth to improve the AODV protocol in order to control congestion. view of the characteristics of MANET there are other parameters that must be taken into account to control and predict congestion.

\section{Proposed Method}

The focus of this work, we present a new enhancement technique for improving QoS in MANET.

Our approach comprises three main building blocks as follows:

1. In order to ensure an efficient diagnosis of packet loss and for a relevant solution we proposed a modification based on Cross layer to collect a succession of the MAC standard IEEE 802.11 TCP Reno at different mobility modes.

2. We proposed a new algorithm based on fuzzy logic that uses stability, energy and signal strength to predict packet losses.

3. In the last process of our contribution, we have compared the three types of protocols OLSR, DSDV and AODV considering multiple QoS metric to assess interaction with the new TCP contributed.

\subsection{The signal strength}

TCP justify packet losses through congestion and resend packets until they successfully arrive at the receiver. Even if the assumption is assured of the reception of traffic, it lifts the transmission delay. This is why we must predict the cause of packet loss. The authors in [24] proposed to reduce the number of messages regarding link failures based on signal strength. Indeed, in MANETs several parameters enter into packet losses. 


\subsection{Stability}

In Ad hoc Networks all the nodes can move randomly and at any time. The notion of stability that we present in this contribution is based on statistics collected by a node on its neighbor to estimate the durability of the connection. The stability calculation is based on the traffic sent by the emitter $\mathrm{E}$ to the receiver $\mathrm{R}$ as shown in figure 1 . The estimate of this stability is calculated using Bienaymé-Chebyshev inequality [24] as follows in Equation 1:

$$
\mathrm{St}_{\mathrm{ER}}=\left(\sum_{i} \frac{X_{E i}^{2}}{n}\right)-\left(\sum_{i} \frac{X_{E i}}{n}\right)^{2}
$$

Where $\mathrm{X}$ i i: the values of the signal power received from the neighboring at different intervals of time.

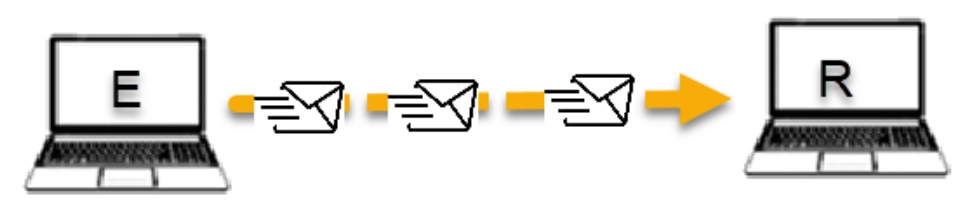

Fig. 1. Archiving process for stability calculation

\section{$4 \quad$ RTT Method and Energy}

In our approach we have used the technique proposed in the routing protocol supporting energy recovery [26] in order to use it in our Fuzzy Logic System. Another way, we have proposed a cross-layer based on RTT solution in order to take into account the particularity of Manet networks and its protocols. Indeed, we used signal strength to identify the distance between nodes. The approach used to estimate the RTT value and compare it with the value of RTT calculated in the normal case to complete the packet loss prediction algorithm as illustrated in the figure 2 by the following Equation 2:

$$
\operatorname{RTT}(\mathrm{n}+1)=\beta R T T(n)+(1-\beta) M
$$

M: The inevitable time of a segment dismissal the ACK

$\boldsymbol{\beta}$ : The stable value between 0 and 1 , control how fast the RTT is suitable for changes.

RTT (n): The value of RTT issued by last packet dispatched.

RTT $(\mathbf{n}+\mathbf{1})$ : depending on this estimated value of RTT (n) and the experimental value of RTT, TCP RENO will be compared them and take their differences as a variable named DIFF. 


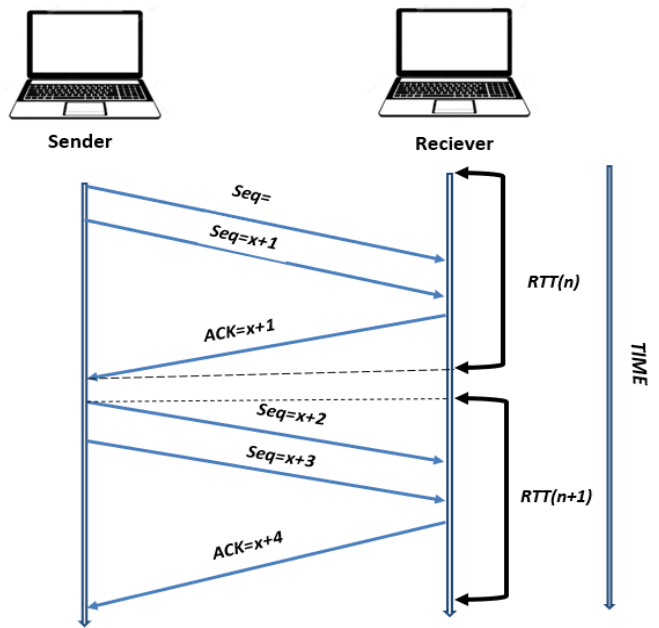

Fig. 2. RTT evaluation scenario and TCP

\subsection{Fuzzy logic structure input crisp for TCP prediction congestion}

In this approach we have proposed Fuzzy Logic to get an algorithm as described below capable of predicting congestion and minimize the overhead for TCP in MANET environment structure. In this regard, we have selected and analysed the main metrics that can cause packet losses. In this approach we have used three metrics: Stability of node, Energy and Signal strength as input crisp in the fuzzy system as shown in figure 3 .

At the process level of the Fuzzification we used Mamdani method as it was described in [27] We have used the Centre of Gravity (COG) [28] as Defuzzification operator for transforming an output MF to a crisp single output decision value (DV) as Equation 3:

$$
D v=F p[S n, E n, S t]
$$

The three inputs of nodes are considered to allow with all possible combinations High, Medium and Low a crisp value defining a quality of node. The three types of values enter nodes allowing us to have 27 possible combinations of imputes. some examples of rules are proposed as follows:

- If the Energy is high, Stability is low and Signal strength is high then the probability of congestion will be high.

- If the Energy is medium, Stability is high and Signal strength is medium then the probability of network congestion will be medium.

- If the Energy is low, Stability is low and Signal strength is high then the probability of network congestion will be low. 


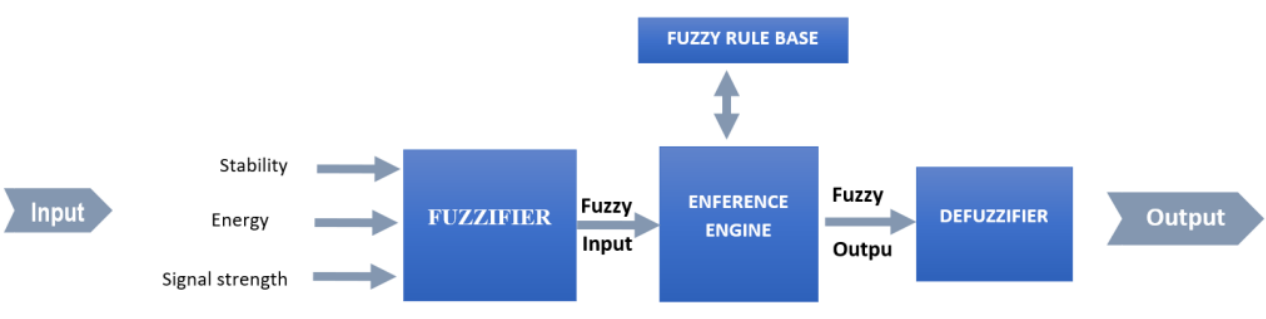

Fig. 3. The general Fuzzy Logic System

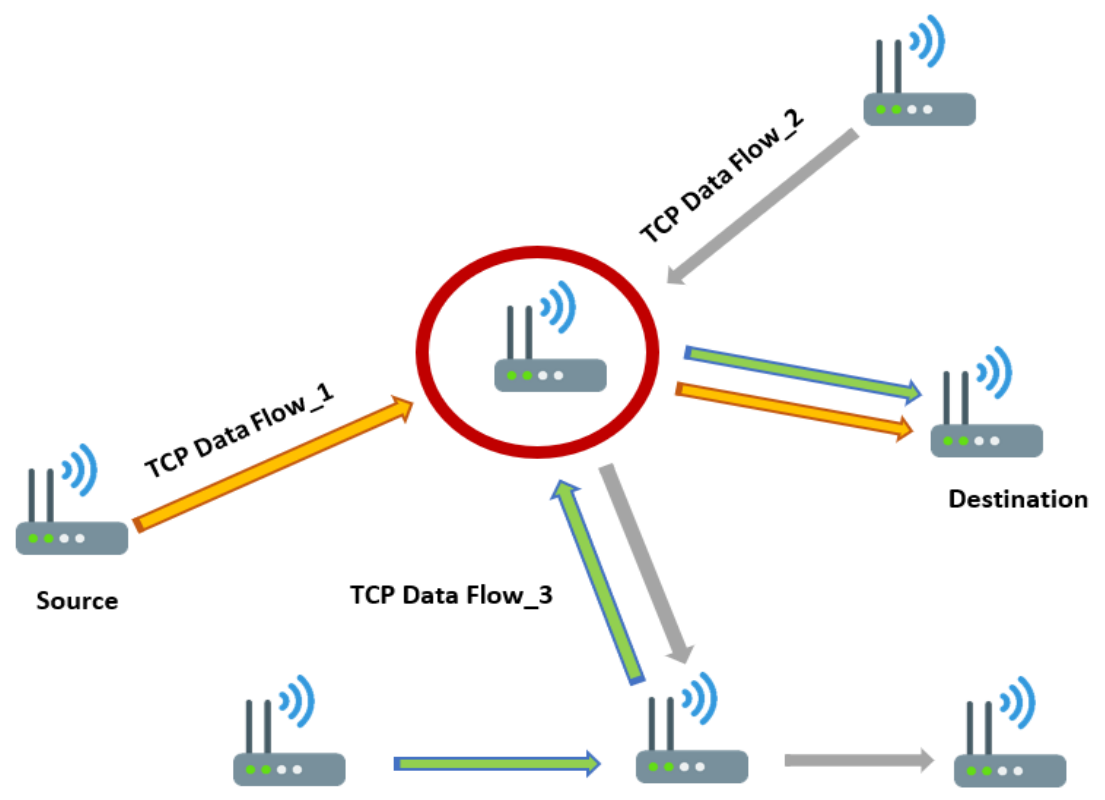

Fig. 4. Scenario described the network interference 


\section{Algorithm 1. New Fuzzy HYBRID TCP algorithm:}

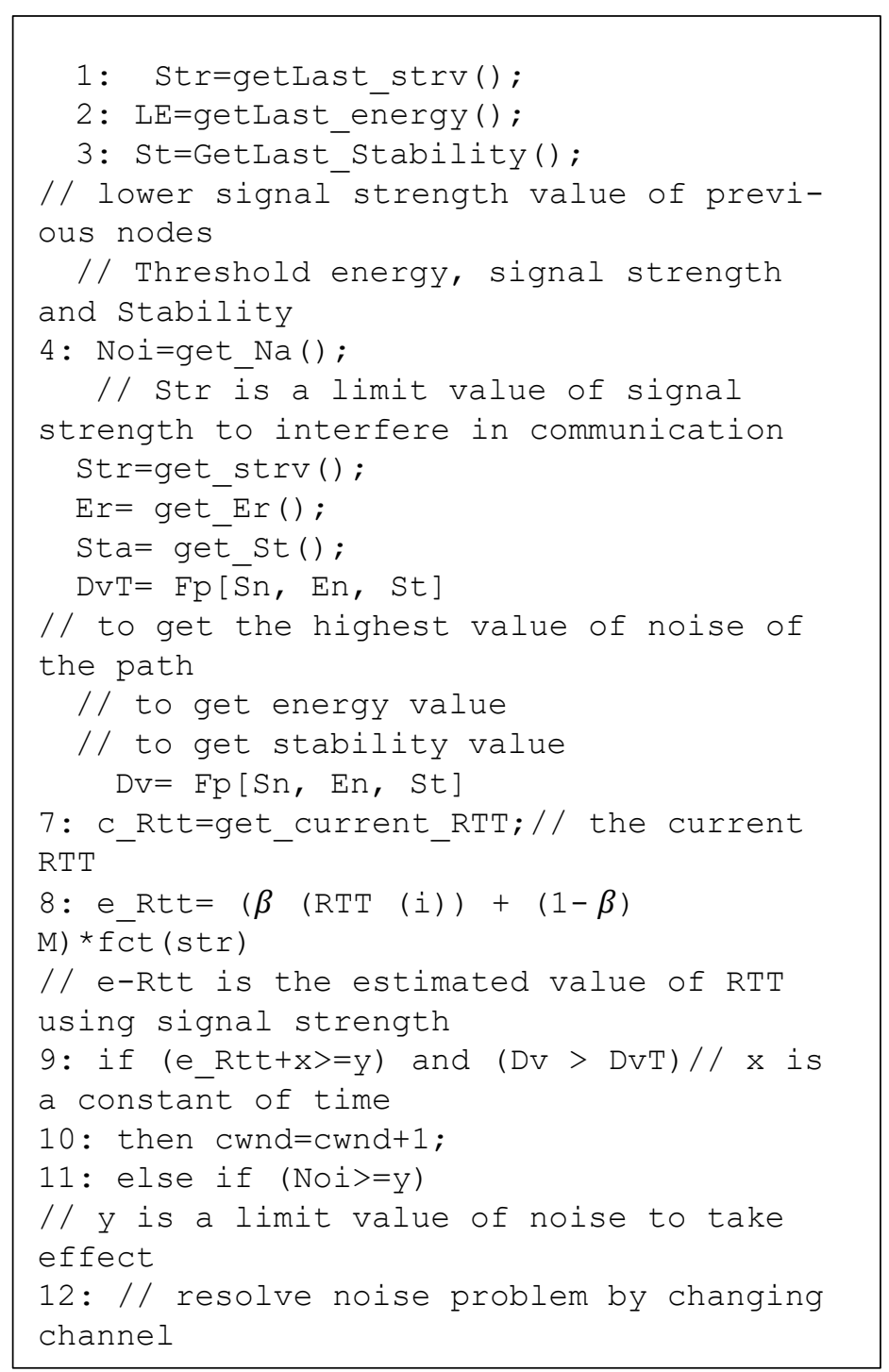

\section{$5 \quad$ Results and Discussions}

In this section, we present the simulation results conducted by Network Simulator 3 (NS-3) with its large TCP library versions. 
Table 1. Parameters values

\begin{tabular}{|l|l|l|c|}
\hline \multicolumn{1}{|c|}{ Parameter } & \multicolumn{1}{c|}{ Value } & \multicolumn{1}{c|}{ Parameter } & Value \\
\hline Channel type & Wireless Channel & Receiving Power & $1.4 \mathrm{w}$ \\
\hline MAC & 802.11 & Idle Power & $1.1 \mathrm{w}$ \\
\hline Routing Protocol & OLSR, DSDV, AODV & Sleep Power & $0.002 \mathrm{w}$ \\
\hline Speed & $10,20,30,40,50 \mathrm{~m} / \mathrm{s}$ & TC-INTERVAL & $5 \mathrm{sec}$ \\
\hline Transmissionn Range & $250 \mathrm{~m}$ & Hello- INTERVAL & $3 \mathrm{sec}$ \\
\hline Initial Energy & $100 \mathrm{Jouble}$ & Topography & $1000 \mathrm{mX} 1000 \mathrm{~m}$ \\
\hline Transmission Power & $1.65 \mathrm{w}$ & Time of simulation & $300 \mathrm{Sec}$ \\
\hline
\end{tabular}

We have chosen the parameters cited in table 1, and as shown in the figure 4 we have created a network congestion based on six nodes referenced to [29].

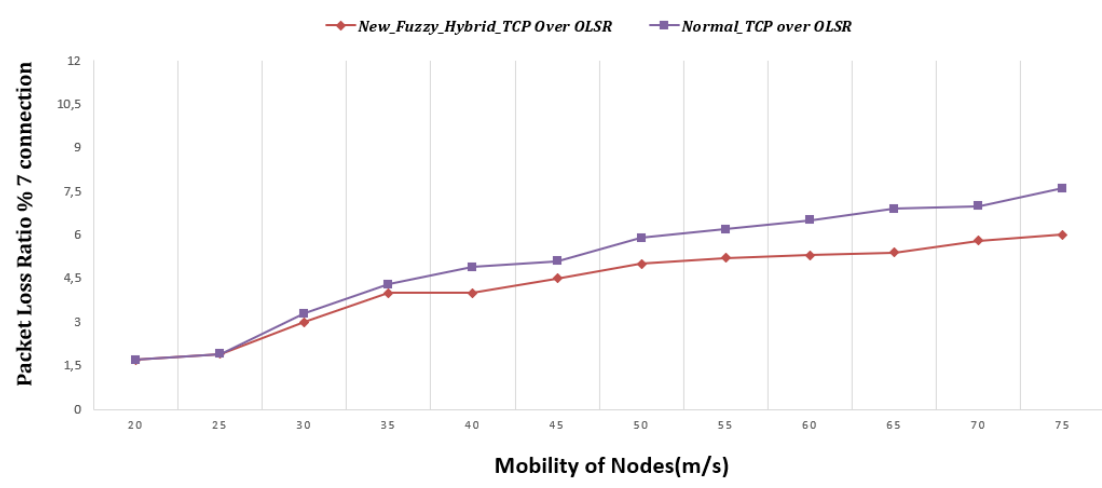

Fig. 5. Packet loss rate of New Fuzzy hybrid TCP over OLSR

In our simulation result, we measured the number of packets lost as a function of different mobility of nodes. The results obtained showed that our protocol gives good results for the various protocols as shown in the figure 5 for OLSR. The analysis of the graph (figure 5) comparative simulation scenario shows that: If we are in a wireless environment with a mobility of less than $25 \mathrm{~m} / \mathrm{s}$, the nodes of the latter consider neither mobile nor under the effect of interference, the three protocols tested (with a congestion control mechanism) give almost the same results in terms of delivered throughput. The explanation is that in a case considered ideal, a wireless environment can be thought of as a wired environment (where packet loss is mainly due to congestion).

Once the mobility exceeds $25 \mathrm{~m} / \mathrm{s}$, we notice that the removal of the node from the coverage area of the wireless environment negatively impacts the signal strength of that node. This effect is reflected in the TCP packet loss mechanism. 


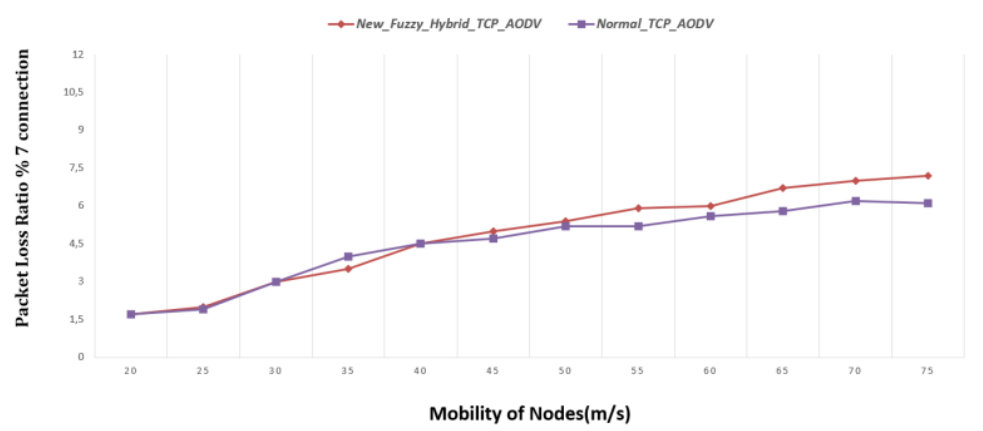

Fig. 6. Packet loss rate of New Fuzzy hybrid TCP over AODV

In the figures 6 and 7 to study the case respectively AODV and DSDV, this was noticed once exceeding the mobility of $25 \mathrm{~m} / \mathrm{s}$. These results are due to the influence of the new improvement on TCP which considers the trade-off between the different metrics having a direct or indirect impact on congestion. Also, the mobility measure which distinguishes the ad hoc network by the stability metric.

The New Fuzzy Hybrid TCP improves the throughput as shown in the figures 8, 9 and 10. This result is due to the fact that New Fuzzy Hybrid TCP takes into consideration the mobility of the nodes, on the other side normal TCP considers the mobility as congestive which degrades Throughput.

As our approach is also based on signal strength, the results are quite close to those of the OLSR protocol. The reason is that once the signal strength starts to weaken and causes some packet loss, our approach disables the TCP stream window reduction mechanism, which leads to the continuation of the transfer (new packets or lost packets) with high speed.

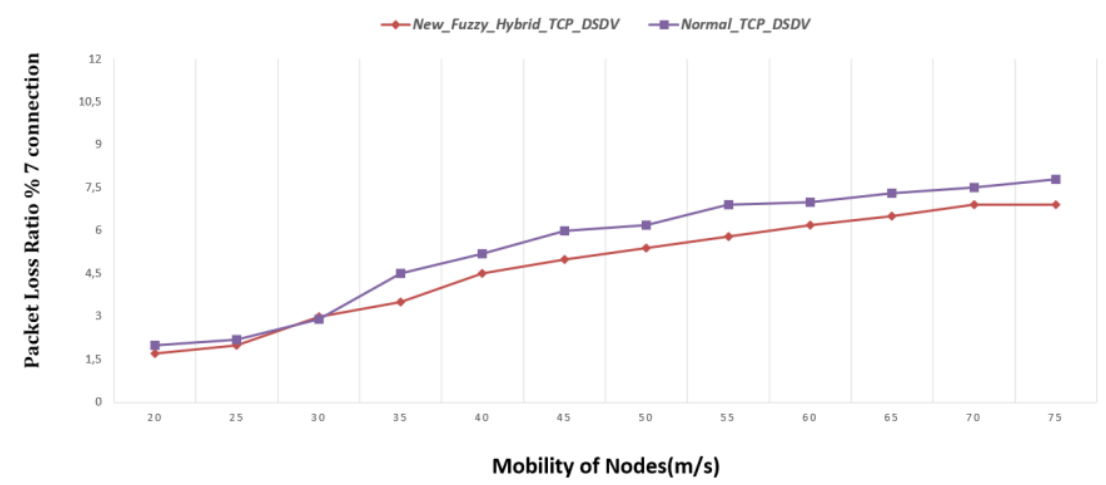

Fig. 7. Packet loss rate of New Fuzzy hybrid TCP and Normal TCP over DSDV 


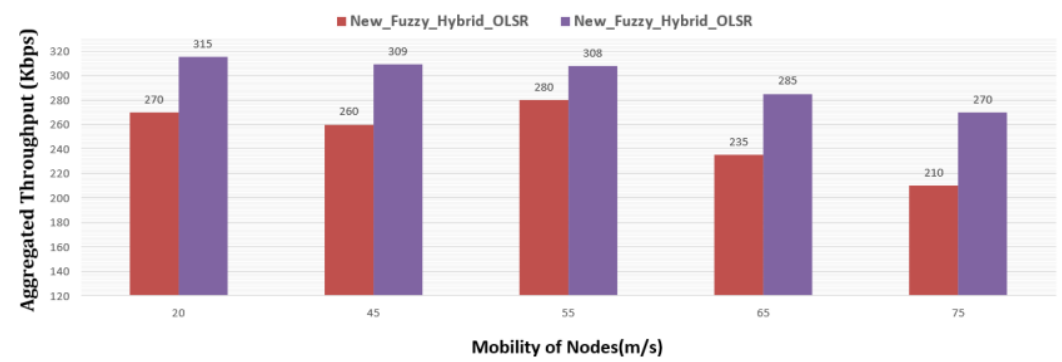

Fig. 8. Throughput Measured of New Fuzzy hybrid TCP and Normal TCP over OLSR

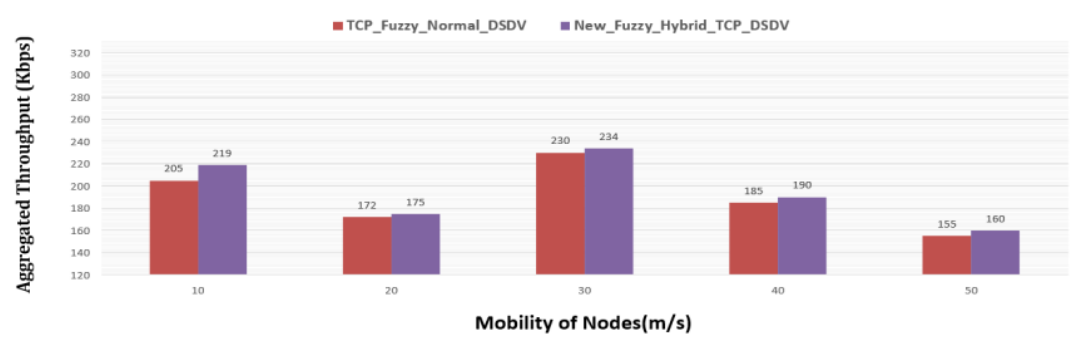

Fig. 9. Throughput Measured of New Fuzzy hybrid TCP and Normal TCP over DSDV

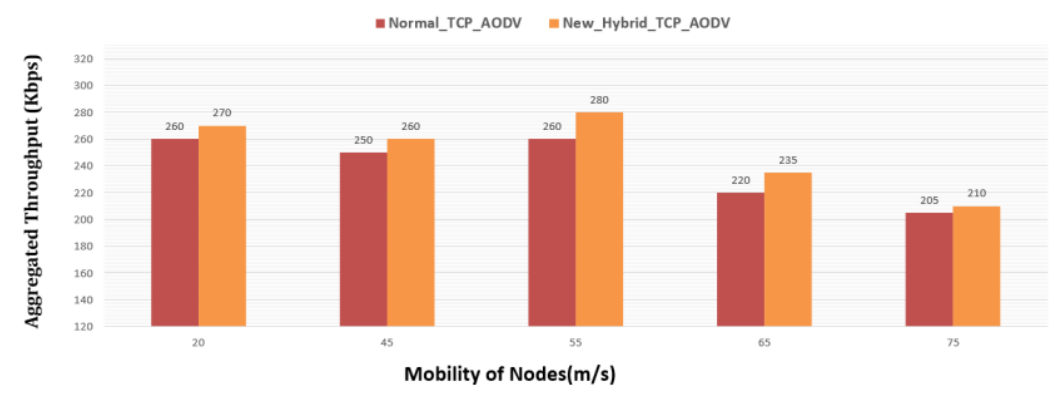

Fig. 10.Throughput Measured of New Fuzzy hybrid TCP and Normal TCP over AODV

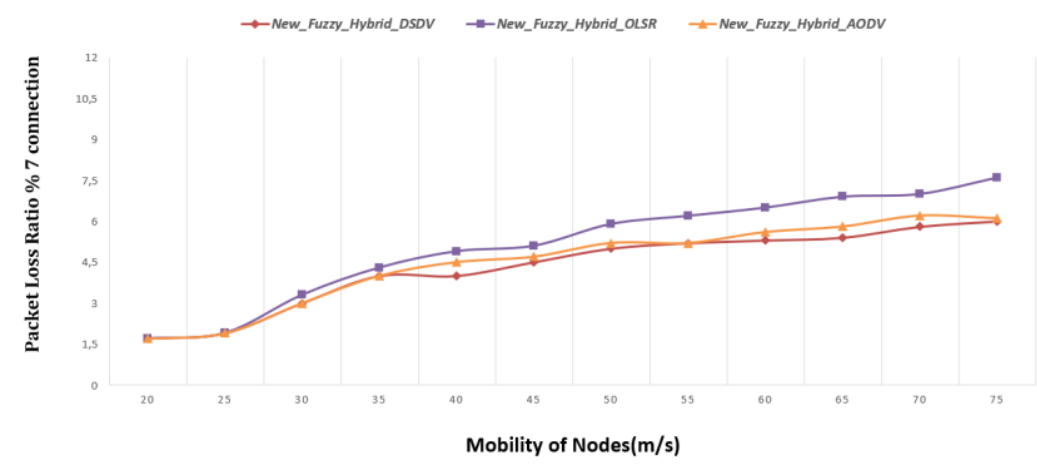

Fig. 11.The comparison between OLSR, AODV and DSDV in case of packet loss 


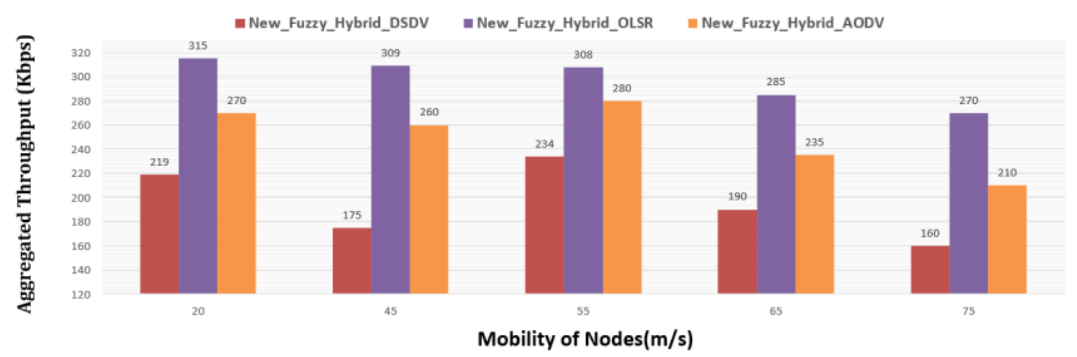

Fig. 12.Throughput Measured over OLSR, AODV and DSDV with mobility node

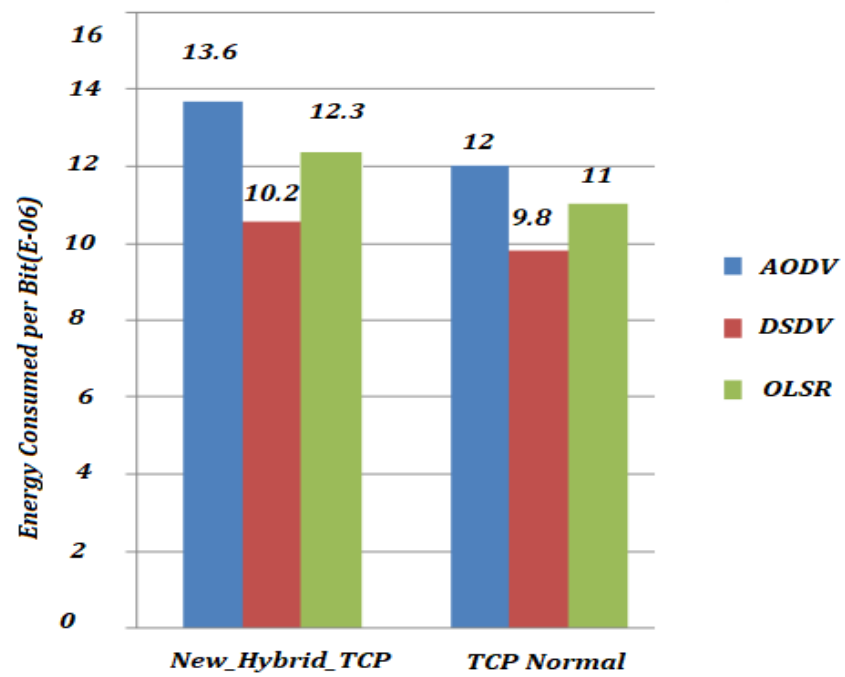

Fig. 13.The comparison between OLSR, AODV and DSDV in case of energy consumed per received bit

In the second experimental part of our contribution, we consider the static MANET environment without changing the route in order to differentiate the effect of interference from other reasons for packet loss.

To select the most efficient and adequate protocol for our New_Fuzzy_hybrid_TCP we compared the protocols in terms of Packet Loss Ratio, Aggregate Throughput and Energy Consumed. We noticed that the OLSR protocol set a very low packet loss level as shown in figure 11 and gives good results in terms of throughput as shown in the figure 12 compared to AODV and DSDV, and this was noticed once exceeding the mobility of $30 \mathrm{~m} / \mathrm{s}$. This result explains the main features of OLSR that use the Multi-Point Relays to route packets. On the other hand, the figures 13 show that the protocols OLSR and AODV consume more energy than AODV over NEW Fuzzy Hybrid TCP. due to increased routing overhead to route maintenance. 
On the other hand, in our approach New_Fuzzy_hybrid_TCP considers the intensities of the noise and changes the channel to have better performance when the noise is average. When the intensity of the latter reaches a high enough value, TCP deactivates the flow reduction mechanism and continues to transmit at the same rate. This justifies the good results of our approach as shown in figure 28.

\section{Conclusion}

In this manuscript, we improve the congestion control mechanism by adding new features that allow it to distinguish between packet loss due to congestion and those due to the MANET environment. For this we called on the link layer through a crosslayer solution to recover the signal power, stability and energy values which will serve as packet loss type indicators for the congestion control mechanism. and we used fuzzy logic to retrieve the metric values in approximative form. Therefore, activate and deactivate it depending on the type of loss. Our solution has been tested in different scenarios with different conditions. The results obtained were compared with different routing protocols (OLSR, DSDV, and AODV) under the same conditions. After comparison, our approach met our expectations. Packet loss due to the wireless environment was handled well and TCP was able to distinguish it from congestion loss. However, although we were satisfied with a distinction of packet loss.

As a perspective of this work, it would be interesting to modify our approach to implement it in a real case (emulation) by trying to predict the signal power and noise values of the current communication from the previous communications. In this vision, the solution will avoid doing the interlayer on the same packet, since the values that will be used will be retrieved from previous communications.

\section{$7 \quad$ References}

[1] D. C. Dobhal and S. C. Dimri, "Performance evaluation of proposed-TCP in Mobile Ad Hoc Networks (MANETs)," 2016 International Conference on Inventive Computation Technologies (ICICT), Coimbatore, 2016, pp. 1-6, https://doi.org/10.1109/inventive. $\underline{2016.7824797}$

[2] Geetha Jayakumar, Gopinath Ganapathi, "Reference Point Group Mobility and Random Waypoint Models in Performance Evaluation of MANET Routing Protocols", Journal of Computer Networks and Communications, vol. 2008, Article ID 860364, 10 pages, 2008. https://doi.org/10.1155/2008/860364

[3] Y. Lien and Y. Yu, "Hop-by-Hop TCP over MANET," 2008 IEEE Asia-Pacific Services Computing Conference, Yilan, 2008, pp. 1150-1155, https://doi.org/10.1109/ apscc. 2008.93

[4] B. Singh and R. Hans, "TCP and UDP Based Performance Analysis of AODV, DSR and DSDV Routing Protocols Under Different Traffic Conditions in Mobile AdHoc Networks", International Journal of Future Generation Communication and Networking, Vol. 8, No. 2 (2015), pp. 73-92. https://doi.org/10.14257/ijfgcn.2015.8.2.7 
[5] Yuan-Ying Hsu and Chien-Chao Tseng, "Prime DHCP: a prime numbering address allocation mechanism for MANETs," in IEEE Communications Letters, vol. 9, no. 8, pp. 712714, Aug. 2005, https://doi.org/10.1109/lcomm.2005.1496591

[6] C. Tsai and S. Hsu, "Study of SCTP over Mixed-Traffic Communication Networks," 2006 International Symposium on Communications and Information Technologies, Bangkok, 2006, pp. 222-226. https://doi.org/10.1109/iscit.2006.340035

[7] U. O. Ibom, "TCP Performance over MANET," 2008 International Conference on Information Networking, Busan, 2008, pp. 1-5. https://doi.org/10.1109/icoin. 2008.4472785

[8] Rui Zhao, "Deep Reinforcement Learning Based Mobile Edge Computing for Intelligent Internet of Things," Physical Communication, vol. 43, pp. 101184, 2020.https://doi.org/10. 1016/j.phycom.2020.101184

[9] Ke He, "Ultra-reliable MU-MIMO detector based on deep learning for 5G/B5G-enabled IoT," Physical Communication, vol. 43, pp. 101181, 2020.https://doi.org/10.1016 li.phycom.2020.101181

[10] S. Prabhavat, W. Narongkhachavana, T. Thongthavorn, C. Phankaew, "Low Overhead Localized Routing in Mobile Ad Hoc Networks", Wireless Communications and Mobile Computing, vol. 2019, Article ID 9652481, 15 pages, 2019.https://doi.org/10.1155/2019/ $\underline{9652481}$

[11] M H. Hanin, M. Amnai, Y .Fakhri, New adaptation method based on cross layer and TCP over protocols to improve QoS in mobile ad hoc network, Vol 11, No 3, p-ISSN 20888708, e-ISSN 2722-2578. https://doi.org/10.11591/ijece.v11i3.pp2134-2142

[12] Kumar, Josh; Kathirvel, A. Analysis and Ideas for Improved Routing in MANET. International Jour-nal of Interactive Mobile Technologies (iJIM), [S.1.], v. 13, n. 04, p. pp. 164177, Apr. 2019. ISSN 1865-7923https://doi.org/10.3991/ijim. v13i04.9928

[13] RAGAB, Ahmed Refaat Sobhy Ahmed. A New Classification for Ad-Hoc Network. International Journal of Interactive Mobile Technologies (iJIM), v. 14, n. 14, p. pp. 214-223, aug. 2020. ISSN 1865-7923. https://doi.org/10.3991/ijim.v14i14.14871

[14] Tao Wang, William N. N. Hung, "Reliable Node Clustering for Mobile Ad Hoc Networks", Journal of Applied Mathematics, vol. 2013, Article ID 285967, 8 pages, 2013. https://doi.org/10.1155/2013/285967

[15] W. Zheng, Y.Li ,X.Jing, and S.Liu1, Adaptive Finite-Time Congestion Control for Uncertain TCP/AQM Network with Unknown Hysteresis, 2020, SN: 1076-2787, https://doi.org/10.1155/2020/4138390

[16] Mohammad Al Mojamed, "Integrating Mobile Ad Hoc Networks with the Internet Based on OLSR", Wireless Communications and Mobile Computing, vol. 2020, Article ID 8810761, 16 pages, 2020. https://doi.org/10.1155/2020/8810761

[17] R. U. Zaman, K. u. R. Khan, M. A. Waseem, A. Farokhi and A. V. Reddy, "Traffic priority-based gateway selection in Integrated Internet-MANET," 2016 2nd International Conference on Applied and Theoretical Computing and Communication Technology (iCATccT), Bangalore, 2016, pp. 18-21, https://doi.org/10.1109/icatcct. 2016.7911958

[18] S. Anbu Karuppusamy, K. Batri, «Improving the performance of TCP in Ad hoc networks based on signal strength and buffering system», Journal of Applied Sciences Research, 8(5): 2554-2563, 2012.

[19] Suveg Moudgil and Dr. Sanjeev Rana "Cross layer Based Synchronized Congestion Control Scheme for Improving TCP Performance" International Journal of Computer Science and Information Security (IJCSIS), Vol. 14, No. 9, September 2016.

[20] C. Mbarushimana, A. Shahrabi, Alleviating contention-induced spurious timeouts in QoSaware MANETs, Ad Hoc Networks, Volume 11, Issue 3,2013, Pages 879-893, ISSN 15708705. https://doi.org/10.1016/j.adhoc.2012.09.009 
[21] Dimitris Kanellopoulos, Congestion control for MANETs: An overview, ICT Express, Volume 5, Issue 2, 2019, Pages 77-83, ISSN 2405-9595. https://doi.org/10.1016/j.icte. 2018.06.001

[22] Dazhong Wang, Shujing Wu, "Design of the Congestion Control for TCP/AQM Network with Time-Delay", Mathematical Problems in Engineering, vol. 2014, Article ID 834698, 7 pages, 2014. https://doi.org/10.1155/2014/834698

[23] Sirajuddin M.D., Rupa C., Prasad A. (2016) Advanced Congestion Control Techniques for MANET. In: Satapathy S., Mandal J., Udgata S., Bhateja V. (eds) Information Systems Design and Intelligent Applications. Advances in Intelligent Systems and Computing, vol 433. Springer, New Delhi. https://doi.org/10.1007/978-81-322-2755-7 28

[24] S. Anbu Karuppusamy, K. Batri, «Improving the performance of TCP in Ad hoc networks based on signal strength and buffering system», Journal of Applied Sciences Research, 8(5): 2554-2563, 2012.

[25] Csiszar V, F-Mori T. A Bienayme-Chebyshev inequality for scale mixtures of the multivariate normal distribution. Mathematical Inequalities and Applications 2009;12(4):83944. https://doi.org/10.7153/mia-12-67

[26] Q. Tan, W. An, Y. Han, Y. Liu, S. Ci, F.-M. Shao, and H. Tang, "Energy harvesting aware topology control with power adaptation in wireless sensor networks," Ad Hoc Networks, vol. 27, pp. 44 - 56, 2015. https://doi.org/10.1016/j.adhoc.2014.11.022

[27] E. H. Mamdani and S. Assilian, An experiment in linguistic synthesis with a fuzzy logic controller, International Journal of Man-Machine Studies, 7(1):1-13,1975. https://doi.org/10.1016/s0020-7373(75)80002-2

[28] S. N. Sivanandam, S. Sumathi and S. N. Deepa, (2007) 'Introduction to fuzzy logic using MATLAB' Springer-Verlag Berlin Heidelberg, New York, ISBN-10 3-540-357807.https://doi.org/10.1007/978-3-540-35781-0

[29] Sofiane Hamrioui, Pascal Lorenz, Jaime Lloret, Joel J.P.C. Rodrigues, Mustapha Lalam, Chapter 2 - Network planni nng and designing, Editor(s): Mohammad S. Obaidat, Petros Nicopolitidis, Faouzi Zarai, Modeling and Simulation of Computer Networks and Systems, Morgan Kaufmann, 2015, Pages 33-53, ISBN 9780128008874. https://doi.org/10.1016/ b978-0-12-800887-4.00002-x

\section{Authors}

Moulay Hicham Hanin is currently a Ph.D. Researcher in optimization service quality of AD-HOC networks within the Computer Science laboratory, department of computer science, Ibn Tofail University, Morocco. Email: moulay.hicham.hanin@ uit.ac.ma

Mohamed Amnai is currently Professor at department of computer science, Ibn Tofail University, Kenitra, Morocco. His main research interest is related to wireless mobile networks network. Email: mohamed.amnai@uit.ac.ma

Fakhri Youssef is currently Professor at department of computer science, Ibn Tofail University, Kenitra, Morocco. His main research interest is related to wireless mobile networks network. Email: fakhri@uit.ac.ma

Article submitted 2021-02-13. Resubmitted 2021-04-08. Final acceptance 2021-04-30. Final version published as submitted by the authors. 